\title{
The Photometric Study of Neglected Short-Period Eclipsing Binary BS Vulpeculae
}

\author{
Miloslav Zejda ${ }^{1}$, Zdeněk Mikulášek ${ }^{1,2}$, Liying $\mathbf{Z h u}^{3,4}$, \\ Shengbang Qian ${ }^{3,4}$, and Jiří Liška ${ }^{1}$
}

\footnotetext{
${ }^{1}$ Department of Theoretical Physics and Astrophysics, Masaryk University, CZ-611 37 Brno, Czech Republic, e-mail: zejda@physics.muni.cz, mikulas@physics.muni.cz

${ }^{2}$ VŠB - Technical Univ., Observatory and Planetarium of J. Palisa, Ostrava, Czech Rep.

${ }^{3}$ National Astronomical Observatories/Yunnan Observatory, Chinese Academy of Sciences, P.O. Box 110, 650011 Kunming, China, e-mail: zhuly@ynao.ac.cn, qsb@ynao.ac.cn

${ }^{4}$ Key Laboratory for the Structure and Evolution of Celestial Objects, Chinese Academy of Sciences P.O. Box 110, 650011 Kunming, China
}

\begin{abstract}
The preliminary results of a study of a neglected, relatively bright, short-periodic $(P=0.48 \mathrm{~d})$, near contact eclipsing binary BS Vulpeculae is given. We present our new complete ( $B$ VRI) light curves, and physical parameters of the system based on them, derived by the 2003 version of the Wilson-Van Hamme code.
\end{abstract}

Keywords. stars: binaries: eclipsing, stars: fundamental parameters, stars: individual BS Vul

\section{Introduction}

The light variations of BS Vul ( $\left.\alpha=19^{\mathrm{h}} 37^{\mathrm{m}} 27^{\mathrm{s}}, \delta=+21^{\circ} 55^{\prime} 50^{\prime \prime}, 2000.0\right)$ were revealed in 1928. Shaw (1994) included this star in his list of near contact binaries and gives the spectral type F2, apparent magnitude $m_{\mathrm{V}}=11 \mathrm{mag}$ with depths of minima 0.7 and 0.2 mag respectively, and the distance of the system as $460 \mathrm{pc}$. We present the first detailed study of this quite bright, but still neglected eclipsing binary.

\section{Observational data and photometric solution}

Usually, only data in the vicinity of brightness minima are used for the period analysis. However, to utilize all available information in the entire light curve, we collected as many individual measurements as possible.

Our own CCD measurements were obtained in 4 nights in the autumn of 2009 and another 4 nights in the autumn 2010. We used an 85cm telescope at the Xinglong Station of National Astronomical Observatory, China. We supplemented the observations with $V$ measurements from de Bernardi \& Scaltriti (1979), ASAS-3, OMC INTEGRAL and the AAVSO archive, thus we collected and used 7959 measurements for the preliminary period analysis and to determine possible changes in the period. We found the following new light ephemeris:

$$
\text { MinJDH }=2452500.16969(7)+0.475970417(10) \times E
$$

Our $B V(I R)_{c}$ CCD light curves were analyzed using the 2003 version of the WilsonVan Hamme code (Wilson \& Van Hamme 2003). Given the F2 spectral type of BS Vul, we assumed an effective temperature of $T_{1}=7000 \mathrm{~K}$ for the primary component (the star eclipsed at primary minimum). We took the gravity-darkening coefficients as $g_{1}=g_{2}=0.32$ and the bolometric albedo as $A_{1}=A_{2}=0.5$, corresponding to the convective envelope of this binary system. Bolometric and bandpass square-root limb-darkening parameters were taken from Van Hamme's paper (1993). 


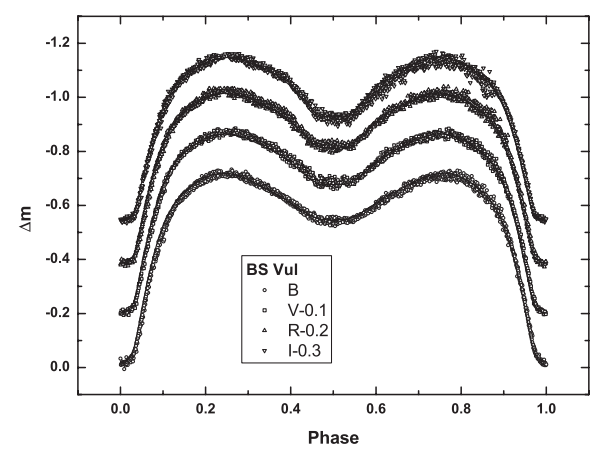

Figure 1. Observed and theoretical $B V(I R)_{c}$ light curves of BS Vul.

Table 1. Photometric solutions of BS Vul

\begin{tabular}{cccccc}
\hline$i(\mathrm{deg})$ & $88.4(9)$ & $r_{1}$ (pole) & $0.4459(7)$ & $\frac{L_{1 B}}{L_{1 B_{1}}+L_{2 B}}$ & $0.9761(3)$ \\
$T_{1}(\mathrm{~K})$ & 7000 & $r_{1}$ (side) & $0.4777(8)$ & $\frac{L_{1 I}}{L_{1} L_{1 V}^{+L_{2 I}}}$ & $0.9202(3)$ \\
$T_{2}(\mathrm{~K})$ & $4644(18)$ & $r_{1}$ (back) & $0.5036(8)$ & $\frac{L_{1 V}}{L_{1 V_{1 R}+L_{2 V}}}$ & $0.9560(3)$ \\
$q$ & $0.340(3)$ & $r_{2}$ (pole) & $0.2700(32)$ & $\frac{L_{1 R}}{L_{1 R}+L_{2 R}}$ & $0.9452(3)$ \\
$\Omega_{1}$ & 2.5530 & $r_{2}$ (side) & $0.2812(38)$ & & \\
$\Omega_{2}$ & $2.5549(13)$ & $r_{2}$ (back) & $0.3136(62)$ & & \\
\hline
\end{tabular}

After solving for the mass ratio $q$, the solutions for several assumed values of mass ratio were obtained. For each $q$, the calculation started at mode 2 (the detached mode). The minimum $\Sigma$ was achieved at $q=0.34$ for BS Vul with mode 4 (the semidetached case with the primary component filling the critical Roche Lobe). Therefore we performed a differential correction so that it converged by making $q$ an adjustable parameter and by choosing $q=0.34$ as the initial value. Finally the converged photometric solutions listed in Table 1 were obtained. The theoretical light curves computed with these values are plotted in Fig. 1 with solid lines. Our solution indicates that BS Vul is a near contact binary system. The primary component fills its critical Roche Lobe and the secondary one nearly fills its Roche lobe too.

\section{Conclusions}

BS Vul belongs to the interesting group of near contact binaries. We determined the parameters of the system. However, for further study spectroscopic observations are highly desired. Continued photometric monitoring and in-depth inspection of all published data are necessary for the description of possible period changes.

\section{Acknowledgements}

This study was partly supported by grants GAAV IAA 301630901, GAČR 205/08/ 0003, MUNI/A/0968/2009, and MŠMT ME10099 and by the West Light Foundation of The Chinese Academy of Sciences, Chinese Natural Science Foundation (Nos. 10973037 and 10903026).

\section{References}

de Bernardi, C. \& Scaltriti, F. 1979, A\&AS, 35, 63

Shaw, J. S. 1994, MemSAI, 65, 95

Van Hamme W., 1993, AJ, 106, 2096

Wilson R. E. \& Van Hamme W., 2003, Computing Binary Stars Observables, the 4th edition of the W-D program., Ftp: astro. ufl. edu, directory pub /wilson/lcdc2003 